\title{
Impact de l'épandage de déchets urbains sur les communautés bactériennes de sols agricoles dans la périphérie de Ouagadougou, Burkina Faso
}

\author{
E. HIEN ${ }^{1}$, S. FAVRE-BONTE ${ }^{2}$, D. MASSE $^{3}$ et S. NAZARET ${ }^{2}$ \\ ${ }^{1}$ Université de Ouagadougou, UFR /SVT 03 BP 7021 Ouagadougou 03,Burkina Faso. \\ ${ }^{2}$ UMR CNRS 5557 Ecologie microbienne, UCB LYON1, 69622 Villeurbanne, France \\ ${ }^{3}$ IRD LEMSAT IRD-ISRA-UCAD Campus Bel-Air B.P. 1386 CP 18524, Dakar-Sénégal \\ *Auteur correspondant ; E-mail: edmond.hien@ird.fr; Tel + 22670263636. Fax + (226) 50310385.
}

\section{RESUME}

Les objectifs de cette étude sont d'évaluer l'influence de certaines activités anthropiques comme l'épandage de déchets urbains sur des terres agricoles qui sont susceptibles de favoriser l'émergence et/ou la dissémination d'agents pathogènes. L'évaluation de l'influence de l'épandage de déchets urbains bruts sur les communautés bactériennes des sols et l'abondance de bactéries pathogènes opportunistes de l'homme a été réalisée. Des sols amendés ou non (Témoin) en déchets ont été échantillonnés sur trois sites de la zone périurbaine de Ouagadougou (Burkina Faso). L'impact sur la communauté bactérienne totale indigène et le risque de dissémination de pathogènes a été évalué par des dénombrements de bactéries cultivables (microflore hétérotrophe cultivable, bactéries indicatrices de contamination fécale et espèces pathogènes de l'homme), ainsi que par une analyse de la structure génétique de la communauté bactérienne par une approche indépendante de la culture (méthode ARISA pour Automated Ribosomal Intergenic Spacer Analysis). Les résultats montrent un enrichissement en bactéries totales cultivables et une modification de la structure génétique des communautés bactériennes dans les parcelles amendées. L'absence de détection de pathogènes tels que les entérocoques et coliformes fécaux, Staphylococcus aureus et pathogènes opportunistes de l'homme (Pseudomonas aeruginosa) suggèrent que les amendements ne sont pas sources de ces pathogènes et ne les sélectionnent pas au sein des communautés. Cependant, le suivi des populations de l'espèce Stenotrophomonas maltophilia, pathogène opportuniste de l'homme fréquemment retrouvé dans les sols, a révélé l'enrichissement de ces populations dans les parcelles amendées. Ces travaux montrent que les activités anthropiques d'épandage de déchets sur des terres agricoles pourraient présenter des risques sanitaires et environnementaux. (C) 2010 International Formulae Group. All rights reserved.

Mots clés : Déchets urbains, sol, communauté bactérienne, pathogènes opportunistes de l’homme.

\section{INTRODUCTION}

La ville de Ouagadougou (Burkina Faso) produit annuellement plus de 300000 tonnes de déchets solides. La production de ces déchets est de plus en plus croissante et compte tenu de la faible teneur des sols en matière organique, partant de la faiblesse des rendements agricoles, avec une quasi absence des sources traditionnelles de matière organique (résidus culturaux, fumiers), les agriculteurs ont recours depuis quelques décennies à ces déchets pour la fertilisation de leurs champs en zone périurbaine sans traitement. Il est indispensable au Burkina 
Faso, à l'échelle de chaque terroir, de valoriser au mieux toutes les ressources organiques pour une production durable (Hien et al., 2006). Cette utilisation directe des déchets pourrait présenter des risques sanitaires et environnementaux pour les agriculteurs, leurs productions et l'environnement.

Un certain nombre d'activités anthropiques tel que les épandages de déchets organiques sur les terres agricoles, le traitement des eaux usées industrielles et domestiques sont susceptibles de disséminer des pathogènes, bactéries, virus, champignons et protozoaires dans l'environnement. Ainsi, divers pathogènes entériques tels que les bactéries Escherichia coli, Campylobacter jejuni, Yersinia enterolitica, Shigella sp. Salmonella enterica,... peuvent être apportés au sol via l'épandage de boues de station d'épuration (Garrec et al., 2003 ; Schwartzbrod et al., 1989 ; De Luca et al., 1998) ou de déjections de divers animaux d'élevage (Meng and Doyle, 1997; Nightingale et al., 2004). De telles pratiques sont donc des sources potentielles d'introduction et de dispersion de microorganismes pathogènes dans les environnements " sol " et " eau" et présentent des risques de contamination des végétaux, des animaux et de l'homme (Al-Ghazali et al., 1990; Solomon et al. , 2002).

Par ailleurs, ces déchets sont sources de substrats organiques mais également de contaminants type éléments traces métalliques susceptibles de modifier les équilibres au sein de la microflore indigène des sols. Des bactéries pathogènes opportunistes présentes dans ces sols peuvent alors se trouver affectées tant par leur abondance populationnelle que par leur diversité. Ainsi, certaines espèces sont susceptibles de se trouver favorisées par ces nouvelles conditions environnementales. A notre connaissance, aucune étude suffisamment exhaustive ne permet d'apporter des éléments satisfaisants pour répondre aux interrogations sur le niveau de risque de transmission à l'Homme des pathogènes d'origine environnementale ou se développant dans l'environnement en raison de certaines pratiques humaines. Ces populations sontelles virulentes et dans un état physiologique permettant une colonisation efficace d'individus sains ou immuno-déprimés ? Les niveaux populationnels sont-ils suffisants pour permettre cette colonisation?

L'objectif de cette étude est d'évaluer l'influence de l'épandage de déchets organiques urbains sur des terres agricoles en périphérie de Ouagadougou, Burkina Faso, au niveau des microflores bactériennes des sols et plus spécifiquement dans le maintien, le développement et la dispersion de populations de bactéries pathogènes modèles.

\section{MATERIEL ET METHODES}

\section{Sites et stratégie d'échantillonnage}

$\mathrm{Au}$ cours d'une campagne d'échantillonnage réalisée du 14 au 21 juin 2008, des échantillons de sols ont été prélevés sur trois sites (Tabtenga, Toudoubwéogo et Zagtouli) où l'agriculture périurbaine est pratiquée avec l'épandage des déchets urbains. Sur chaque site, les échantillons sont prélevés sur des couples de parcelles amendées ou non (Témoin) en déchets urbains bruts. Sur chaque parcelle, trois transects sont tracés. Sur chaque transect, 10 prélèvements élémentaires de sol distants de $5 \mathrm{~m}$ sont faits. Les prélèvements sont faits dans les couches $0-5 \mathrm{~cm}$ et $5-15 \mathrm{~cm}$. Les 10 échantillons élémentaires par profondeur de chaque transect sont réunis pour former un échantillon composite. Les échantillons sont séchés à l'air, puis tamisés à $2 \mathrm{~mm}$. Pour chaque parcelle, les analyses ont été réalisées en triplicat.

\section{Dénombrements bactériens et identification des isolats}

Les dénombrements de la microflore bactérienne hétérotrophe totale ont été réalisés sur le milieu TSA (Tryptic Soy Agar) dilué au dixième additionné de cycloheximide (200 mg $1^{-1}$ ) pour inhiber la croissance des fonges. Pour cela, $5 \mathrm{~g}$ de sol ont été broyés dans un volume de $50 \mathrm{ml}$ de $\mathrm{NaCl} 0,8 \%$ pendant $90 \mathrm{~s}$ 
dans un Waring Blender (Eberbach Corporation, New Hampshire, USA). Des suspensions/dilutions ont été réalisées dans la solution de $\mathrm{NaCl} 0,8 \%$ et $100 \mu \mathrm{l}$. Des dilutions $10^{-3}$ à $10^{-5}$ ont été étalées sur boîte de Pétri et les colonies ont été dénombrées après 5 jours d'incubation à $28^{\circ} \mathrm{C}$.

L'impact des amendements sur la distribution et l'abondance des populations de bactéries pathogènes a été évalué en ciblant des espèces bactériennes indicatrices de contamination fécale (coliformes, streptocoques/entérocoques), une espèce pathogène primaire de l'homme (Staphylococcus aureus) et 2 espèces pathogènes opportunistes de l'homme Pseudomonas aeruginosa, et Stenotrophomonas maltophilia. Des approches de microbiologie classique à savoir l'isolement sur des milieux de culture sélectifs (milieu favorisant la croissance du microorganisme recherché) ont été utilisées. Ces essais sont couplés à des tests phénotypiques (critères biochimiques évalués par galerie API) et génotypiques (recherche de la présence de gènes marqueurs par PCR en utilisant des couples d'amorces spécifiques de l'espèce et séquençage de l'ADNr 16S) permettant de confirmer l'identité des isolats.

Pour les pathogènes indicateurs, trois milieux (TTC tergitol, Chapman et Slanetz) ont été utilisés et testés sur les échantillons de sol de l'horizon 0-5 cm. Le milieu TTC tergitol permet de rechercher et dénombrer les coliformes. Le tergitol 7 permet de sélectionner les coliformes et le TTC montre le pouvoir réducteur des bactéries. Ainsi, des colonies jaunes sont suspectées d'être apparentées à Escherichia coli ou Enterobacter aerogenes et les colonies rouges sont d'autres coliformes. La gélose Chapman est le milieu sélectif des bactéries halophiles. La présence d'une teneur élevée en $\mathrm{NaCl}$ permet la croissance de bactéries comme Staphylococcus et inhibe celle d'un grand nombre d'espèces. La présence de colonies jaunes entourées d'un halot jaune permet de suspecter la présence de $S$. aureus. La gélose de Slanetz et Bartley est un milieu sélectif utilisé pour le dénombrement des entérocoques intestinaux dans les eaux d'alimentation, les boissons, les eaux usées et divers produits biologiques d'origine animale. La présence d'espèces apparentées au genre Enterococcus (E. faecium et E. faecalis) est indicatrice d'une contamination fécale plutôt d'origine humaine et celle d'espèces apparentées au genre Streptococcus (S. bovis, $S$. equinus ou $S$. gallolyticus) d'une contamination fécale d'origine animale. Les colonies d'intérêt sont rouge foncé à marron. Les dénombrements ont été réalisés comme explicités pour la microflore hétérotrophe totale. Cent $\mu \mathrm{l}$ des dilutions $10^{-1}$ à $10^{-3}$ ont été étalés sur boîte de Pétri et les colonies ont été dénombrées après $24 \mathrm{~h}$ d'incubation à $37^{\circ} \mathrm{C}$.

En ce qui concerne les pathogènes opportunistes, la recherche de l'espèce $P$. aeruginosa a été réalisée sur le milieu $\mathrm{CAB}$ (Cetrimide Agar base, Oxoïd) additionné d'acide nalidixique (15 $\mathrm{mg} \mathrm{l}^{-1}$ ) et de cycloheximide (200 $\mathrm{mg} \mathrm{l}^{-1}$ ) et celle de Stenotrophomonas maltophilia sur le milieu VIA (vancomycin, imipenem, amphotericine B) (Kerr et al., 1996). Cent microlitres des dilutions $10^{-1}$ à $10^{-2}$ ont été étalés sur boite de Pétri et les colonies ont été dénombrées après 24 à $48 \mathrm{~h}$ d'incubation à $37^{\circ} \mathrm{C}$ et à $28^{\circ} \mathrm{C}$ pour P. aeruginosa et à $28^{\circ} \mathrm{C}$ pour S. maltophilia. L'identité des colonies fluorescentes sur CAB est confirmée par une PCR spécifique du marqueur ecfX (Lavenir et al., 2007) et celle sur VIA est confirmée par une PCR spécifique ciblant les gènes smeD et ggpS ( (RibbeckBusch et al., 2005).

\section{Analyse de la structure génétique des communautés bactériennes totales par ARISA}

La méthode ARISA est une approche indépendante de la culture dite d'empreintes génétiques. Cette approche implique une analyse du polymorphisme de longueur des produits d'amplification PCR des intergènes (IGS) entre les gènes rrs (16S) et rrl (23S) dont la taille varie de 50 pb à plus de $1.5 \mathrm{~kb}$ en fonction de l'espèce bactérienne. Les amorces PCR utilisées ciblent les gènes flanquant les 
IGS. Ces amorces sont marquées par des fluorochromes permettant une analyse du polymorphisme par séquenceur automatique. Cette approche a été appliquée sur les ADNs extraits à partir des 36 échantillons de sol.

\section{RESULTATS ET DISCUSSION}

Impact des amendements sur les communautés bactériennes des sols

Les dénombrements de la microflore bactérienne hétérotrophe totale réalisés sur le milieu TSA $1 / 10^{\text {ème }}$ ont mis en évidence des différences d'abondance au niveau du site de Tabtenga entre la zone amendée et la zone non amendée avec une microflore plus importante dans la zone amendée (Figure 1). Une telle différence a été observée sur le site de Toudoubwéogo mais pas sur le site de Zagtouli. Ces observations suggèrent que les amendements sont sources de matières organiques et qu'ils enrichissent les populations hétérotrophes cultivables ou qu'ils facilitent la cultivabilité des cellules. L'ajout de matière organique dans les sols agricoles représente une source d'énergie, de carbone, de nutriments divers pour les microorganismes du sol, d'autant plus favorable que les sols sont pauvres en éléments et limitants pour la croissance des microorganismes. Une augmentation de la biomasse microbienne et du nombre de bactéries hétérotrophes cultivables en réponse à un ajout de matière organique a déjà été rapportée (Sastre et al., 1996; Albiach et al., 2000; Garcia-Gil et al., 2000). Cette augmentation est généralement proportionnelle à la quantité de matière organique apportée (Albiach et al., 2000; Garcia-Gil et al., 2000 ; Saison et al., 2006). Un dénombrement indirect en microscopie permettrait d'évaluer si ces amendements augmentent la biomasse bactérienne. L'absence d'une telle observation dans le site de Zagtouli est soit le reflet d'une absence de stimulation par les apports organiques et/ou celui d'un effet inhibiteur consécutif à la présence d'éléments toxiques (e.g. éléments traces métalliques) dans les déchets (Lejon et al., 2008).
L'analyse de la structure génétique des communautés bactériennes des sols par la méthode ARISA met également en évidence des différences entre parcelles amendées et témoins au sein d'un même site. Il est également observé des différences plus ou moins marquées entre sites et entre horizons d'une même parcelle. Des modifications qualitatives de la microflore bactérienne en réponse à différents types d'amendement ont été observées dans les travaux de Lejon et al. (2007). Ces auteurs mentionnent également que l'amplitude des modifications est dépendante de la nature de l'amendement et des caractéristiques physiques et chimiques des sols.

\section{Impact des amendements sur les espèces pathogènes}

Pour les pathogènes indicateurs, les résultats obtenus sont présentés dans le Tableau 1. Aucune colonie n'a été obtenue sur l'ensemble des échantillons testés sur le milieu de Slanetz démontrant l'absence de bactéries apparentées à Staphylococcus aureus ni dans les sols, ni apportés par les amendements. Des colonies ont été obtenues sur les milieux Chapman et TTC tergitol. Cependant, l'analyse génétique par séquençage de l'ADNr16S n'a pas permis de mettre en évidence de pathogènes apparentées à Escherichia coli ou Enterobacter aerogenes (pathogènes recherchés sur le milieu TTC tergitol). Seules des espèces apparentées à Enterobacter, sp., Enterobacter vulneris et au genre Pantoea ont été identifiées. Similairement, aucun pathogène de type Entérocoques ou Streptocoques n'a été identifié sur le milieu de Chapman. L'ensemble de ces données mettent en évidence l'absence de populations indicatrices de contamination fécale ou de pathogènes apparentés à $S$. aureus dans les amendements qui persistent dans les sols, ceci dans la limite des seuils de détection imposées par la méthodologie (50 à 100 cellules/g de sol sec).

Nos résultats sur l'espèce pathogène opportuniste $P$. aeruginosa montrent que sur l'ensemble des échantillons analysés, seules 
quelques rares colonies fluorescentes (12 sur les 18 échantillons analysés, horizon $0-5 \mathrm{~cm}$ ) ont été obtenues. L'analyse de ces colonies selon des critères biochimiques et tests génétiques montre qu'une seule des colonies appartient à l'espèce $P$. aeruginosa. Les 11 autres colonies sont apparentées à différentes espèces de Pseudomonas, à savoir P. putida, P. fluorescens ou Pseudomonas sp. Ces résultats confirment la semi-sélectivité du milieu $\mathrm{CAB}$, milieu initialement développé pour le contexte clinique, démontrant ses limites pour l'isolement et la quantification de $P$. aeruginosa à partir d'échantillons environnementaux très complexes sur le plan de la diversité des taxons présents. Cette nondétection de $P$. aeruginosa dans les sols échantillonnés n'est pas tout à fait surprenante. En effet, malgré son qualificatif d'ubiquiste, la présence de $P$. aeruginosa en tant qu'habitant indigène $\mathrm{du}$ sol est controversée. En effet, peu d'études ont mis en évidence la présence de $P$. aeruginosa dans les sols et ces études sont relativement anciennes. Les travaux de Ringen et Drake en 1952, montrent que $P$. aeruginosa n'a pu être isolé que dans 3 des 100 sols testés. En 1974, Green et collaborateurs ont eu plus de succès avec une détection dans $23 \%$ des cas (14 sols positifs sur les 58 sols agricoles californiens testés) (Green, et al., 1974). Notre étude suggère par ailleurs que les amendements ne constituent pas une source exogène de $P$. aeruginosa ou que $P$. aeruginosa ne survit pas et/ou ne se multiplie pas dans ces sols. Ces observations sont en accord avec les études de survie. En effet, la capacité de survie de $P$. aeruginosa a été remise en question suite à l'observation de la mort rapide de souches inoculées dans des microcosmes de sol (Zechman \& Casida, 1982).

Les dénombrements sur le milieu VIA spécifique de Stenotrophomonas maltophilia ont permis l'obtention de colonies dont l'identité a pu être confirmée par les tests avec les marqueurs génétiques (Tableau 1). Il apparaît ainsi que la bactérie $S$. maltophilia est présente sur les 3 sites et ceci aussi bien dans les parcelles témoins que dans les parcelles amendées. Cependant, les effectifs les plus importants sont retrouvés dans des parcelles amendées des sites de Tabtenga et de Zagtouli. L'amendement conduit à une augmentation de 1,8 à 4,5 fois des populations de S. maltophilia (Figure 2).

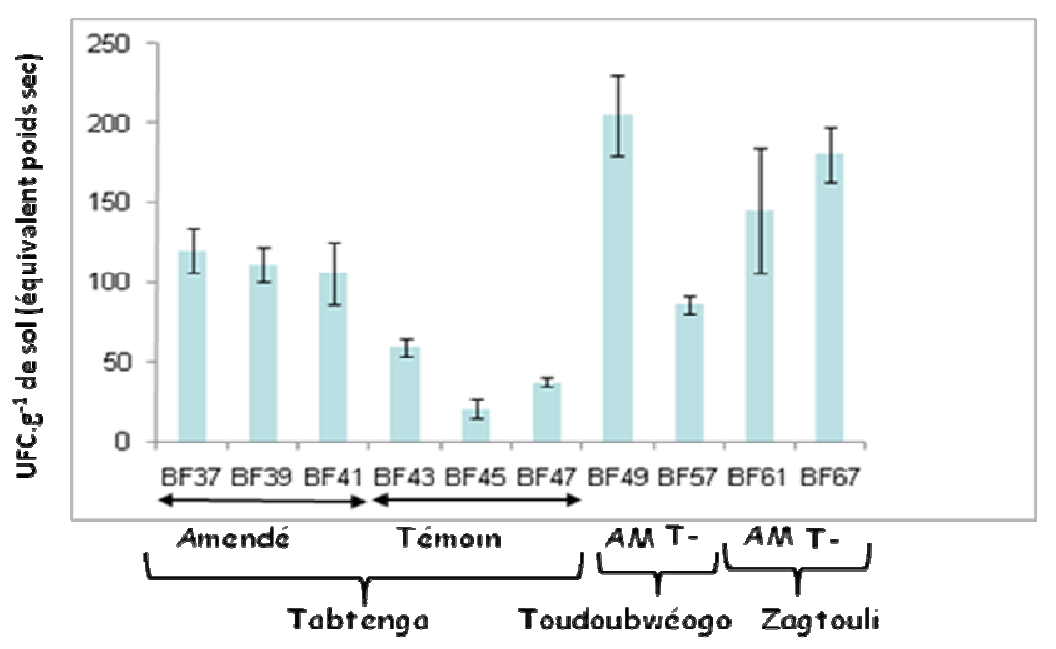

Figure 1 : Dénombrement des bactéries hétérotrophes totales sur le milieu TSA dilué au dixième dans les échantillons de sol de l'horizon 0-5 cm prélevés sur les parcelles amendées et les parcelles non amendées des sites Tabtenga, Toudoubwéogo et Zagtouli au Burkina Faso. Les valeurs représentent les moyennes de 3 répétitions. UFC : Unité Formant Colonie. 
Tableau 1 : Dénombrement des pathogènes dans les échantillons de sol de l'horizon 0-5 cm prélevés sur les parcelles amendées (AM) et les parcelles non amendées (NA) des sites Tabtenga, Toudoubwéogo et Zagtouli au Burkina Faso.

\begin{tabular}{|c|c|c|c|c|c|}
\hline Echantillon & Site & Parcelle & $\begin{array}{l}\text { Chapman } \\
\left(\text { UFC x 10 } \mathbf{1 0}^{5}\right)\end{array}$ & $\begin{array}{l}\text { TTC } \\
\text { tergitol } \\
\left(\text { UFC x 10 } \mathbf{1 0}^{3}\right) \\
\end{array}$ & $\begin{array}{l}\text { VIA } \\
\left(\text { UFC x } 10^{3}\right)\end{array}$ \\
\hline BF3437 & Tabtenga & $\mathrm{AM}$ & 6.51 & 2 & 0 \\
\hline BF3439 & Tabtenga & $\mathrm{AM}$ & 7.7 & 0 & 0 \\
\hline BF3441 & Tabtenga & $\mathrm{AM}$ & 5.2 & 11 & 5.43 \\
\hline BF3443 & Tabtenga & $\mathrm{T}-$ & 0.13 & 0 & 0 \\
\hline BF3445 & Tabtenga & $\mathrm{T}-$ & 0 & 0 & 0 \\
\hline BF3447 & Tabtenga & $\mathrm{T}-$ & 0.47 & 0.333 & 0 \\
\hline BF3449 & Toudoubwéogo & $\mathrm{AM}$ & 13.1 & 2.67 & 0 \\
\hline BF3451 & Toudoubwéogo & $\mathrm{AM}$ & 13.7 & 30 & 0 \\
\hline BF3453 & Toudoubwéogo & $\mathrm{AM}$ & 9.67 & 12 & 1.36 \\
\hline BF3455 & Toudoubwéogo & $\mathrm{T}-$ & 4.43 & 1 & 0 \\
\hline BF3457 & Toudoubwéogo & $\mathrm{T}-$ & 2.2 & 0.33 & 0 \\
\hline BF3459 & Toudoubwéogo & $\mathrm{T}-$ & 2.83 & 1.67 & 0 \\
\hline BF3461 & Zagtouli & $\mathrm{AM}$ & 12.4 & 0 & 0 \\
\hline BF3463 & Zagtouli & $\mathrm{AM}$ & 51.7 & 0 & 0 \\
\hline BF3465 & Zagtouli & $\mathrm{AM}$ & 3.57 & 3.67 & 5.76 \\
\hline BF3467 & Zagtouli & $\mathrm{T}-$ & 3.03 & 1.67 & 0 \\
\hline BF3469 & Zagtouli & T- & 1.7 & 2.33 & 0.669 \\
\hline BF3471 & Zagtouli & T- & 2.5 & 0 & 0.667 \\
\hline
\end{tabular}

Les valeurs représentent les moyennes de 3 répétitions. UFC: Unité Formant Colonie.

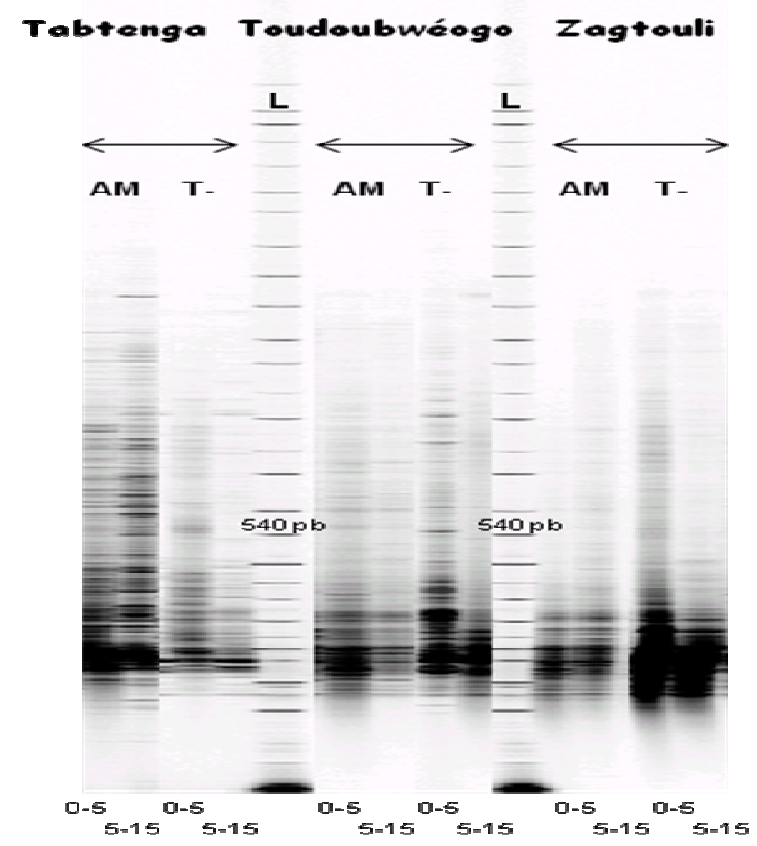

Figure 2 : Profils ARISA des communautés bactériennes des sols des parcelles amendées (AM) et les parcelles non amendées (T-) des sites Tabtenga, Toudoubwéogo et Zagtouli au Burkina Faso. 0-5 et 5-15 correspondent aux 2 horizons analysés. L indique une échelle de poids moléculaire. 


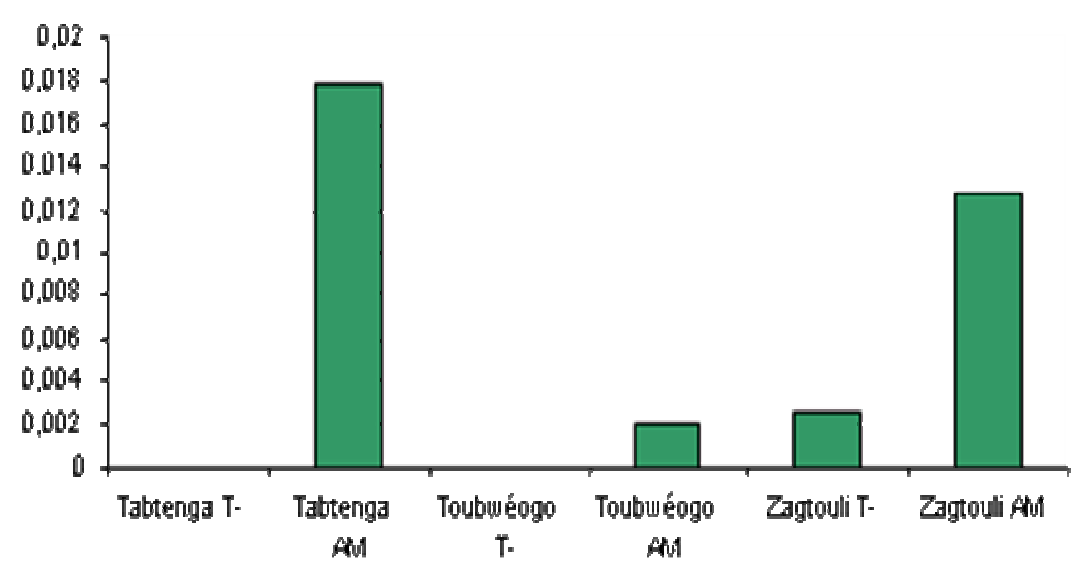

Figure 3 : Pourcentage de $S$. maltophilia au sein de la microflore hétérotrophe cultivable dans les sites amendés (AM) et non amendés (T-) en déchets urbains bruts.

En comparaison, la microflore hétérotrophe cultivable n'augmente que de 2,6 fois dans les sols amendés. Toutefois, il est à noter l'importante variabilité entre les répétitions pour les dénombrements des populations de $S$. maltophilia, variabilité liée à une abondance souvent à la limite des seuils de détection. Cette détection de $S$. maltophilia dans les sols n'est pas tout à fait inattendue car contrairement à $P$. aeruginosa, il est admis que $S$. maltophilia est très répandue dans les sols, qui constituent un des principaux réservoirs de cette bactérie (Ryan et al., 2009). D'abord nommée Pseudomonas maltophilia, puis Xanthomonas maltophilia, S. maltophilia est fréquemment isolé d'environnements terrestres, en particulier de la rhizosphère de différentes plantes (Berg et al., 1996). Toutefois, les études mettant en évidence la prévalence et la densité des populations indigènes du sol de $S$. maltophilia sont rares et les facteurs favorisant sa présence et sa distribution demeurent inconnus.

La présence de $S$. maltophilia dans les sols témoins révèle que cette espèce est indigène de certains sols de la zone périurbaine de Ouagadougou au Burkina Faso. Ses effectifs plus élevés dans les sols amendés peuvent résulter d'un enrichissement consécutif à un apport de matière organique ou d'un apport de populations exogènes présentes dans les amendements (par exemple: présence de souches bactériennes d'origine humaine et/ou hospitalière).

\section{Conclusion}

Ces travaux montrent que les activités anthropiques telles que les pratiques d'épandages de déchets sur des terres agricoles sont susceptibles de favoriser le développement de certaines bactéries pathogènes pouvant présenter des risques sanitaire et environnemental. En effet, par leur richesse en matières organiques, ces amendements accroissent les effectifs de l'ensemble de la communauté bactérienne indigène des sols et de certaines espèces (e.g. S. maltophilia) naturellement présentes dans ces sols. Toutefois, les amendements n'ont pas contribué à disséminer et/ou sélectionner des espèces pathogènes d'origine humaine ou animale.

\section{REFERENCES}

Al-Ghazali MR, Al-Azawi SK. 1990. Listeria monocytogenes contamination of crops grown on soil treated with sewage sludge cake. J. Appl. Bacteriol., 69(5): 642-647.

Albiach R, Canet R, Pomares F, Ingelmo F. 2000. Microbial biomass content and 
enzymatic activities after the application of organic amendments to a horticultural soil. Bioresour. Technol., 75(1): 43-48.

Berg G, Marten P, Ballin G. 1996 Stenotrophomonas maltophilia in the rhizosphere of oilseed rape - Occurrence, characterization and interaction with phytopathogenic fungi. Microbiol. Res., 151(1): 19-27.

De Luca G, Zanetti F, Fateh-Moghadm P, Stampi S. 1998. Occurence of Listeria monocytogenes in sewage sludge. $\mathrm{Zbl}$. Hyg. Unweltmed, 201(3): 269-277

García-Gil JC, Plaza C, Soler-Rovira P, Polo A. 2000. Long-term effects of municipal solid waste compost application on soil enzyme activities and microbial biomass. Soil Biol. Biochem., 32(13): 1907-1913.

Garrec N, Picard-Bonnaud F, Pourcher AM. 2003. Occurrence of Listeria $\mathrm{sp}$ and $L$ monocytogenes in sewage sludge used for land application: effect of dewatering, liming and storage in tank on survival of Listeria species. FEMS Immunol. Med. Microbiol., 35(3): 275-283.

Green SK, Schroth MN, Cho JJ, Kominos SK, Vitanza-jack VB 1974 Agricultural plants and soil as a reservoir for Pseudomonas aeruginosa. Appl Microbiol, 28(6): 987991.

Hien E, Ganry F, Oliver R, 2006. Carbon sequestration in a savannah soil in southwestern Burkina as affected by cropping and cultural practices. Arid Land Res. Manag., 20(2): 133-146.

Kerr KG, Denton M, Todd N, Corps CM, Kumari P, Hawkey PM. 1996. A new selective differential medium for isolation of Stenotrophomonas maltophilia. Eur. J. Clin. Microbiol. Infect. Dis., 15(7): 607610.

Lavenir R, Jocktane D, Laurent F, Nazaret S, Cournoyer B. 2007. Improved reliability of Pseudomonas aeruginosa PCR detection by the use of the species- specific ecfX gene target. J. Microbiol. Meth., 70(1): 20-29.

Lejon DP, Sebastia J, Lamy I, Chaussod R, Ranjard L. 2007. Relationships between soil organic status and microbial community density and genetic structure in two agricultural soils submitted to various types of organic management. Microb. Ecol., 53(4): 650-663.

Lejon DP., Martins JM, Lévêque J, Spadini L, Pascault N, Landry D, Milloux MJ, Nowak V, Chaussod R, Ranjard L. 2008. Copper dynamics and impact on microbial communities in soils of variable organic status. Environ. Sci. Technol., 42(8): 2819-2825.

Meng J, Doyle MP. 1997. Emerging issues in microbiological food safety. Annu. Rev. Nutr., 17(1): 255-275.

Nightingale KK, Schukken YN, Nightingale CR, Fortes ED, Ho AJ, Her Z, Grohn YT, McDonough PL, Wiedmann M. 2004. Ecology and transmission of Listeria monocytogenes infecting ruminants and in the farm environment. Appl. Environ. Microbiol., 70(8): 4458-4467.

Ribbeck-Busch K, Roder A, Hasse D, de Boer W, Martinez JL, Hagemann M., Berg G. 2005 A molecular biological protocol to distinguish potentially human pathogenic Stenotrophomonas maltophilia from plant-associated Stenotrophomonas rhizophila. Environ. Microbiol., 7(11): 1853-1858.

Ringen LM, Drake CH. 1952. A study of the incidence of Pseudomonas aeruginosa from various natural sources. $J$. Bacteriol., 64(6): 841-845.

Ryan RP, Monchy S, Cardinale M, Taghavi S, Crossman L, Avison MB, Berg G, van der Lelie D, Dow JM. 2009. The versatility and adaptation of bacteria from the genus Stenotrophomonas. Nat. Rev. Microbiol., 7(7): 514-25.

Saison C, Degrange V, Oliver R, Millard P, Commeaux C, Montange D, Le Roux X. 
2006. Alteration and resilience of the soil microbial community following compost amendment: effects of compost level and compost-borne microbial community. Environ. Microbiol., 8(2): 247-257.

Sastre I, Vicente MA, Lobo MC. 1996. Influence of the application of sewage sludges on soil microbial activity. Bioresour. Technol., 57(1): 19-23.

Schwartzbrod J, Papadopoulos O, Burdin JC. 1989. Detection and behaviour of Listeria spp. In food and environmental sample by esculin hydrolysis. J. Food Prot., 51(3): 762-765.

Solomon EB, Yaron S, Matthews KR. 2002. Transmission of Escherichia coli O157:H7 from contaminated manure and irrigation water to lettuce plant tissue and its subsequent internalization. Appl. Environ. Microbiol., 68(1): 397-400.

Zechman JM, Casida LE. 1982. Death of Pseudomonas aeruginosa in soil. Can J Microbiol., 28(7): 788-794. 